\title{
Restoration of Noisy Blurred Images Using MFPIA and Discrete Wavelet Transform
}

\author{
Dunia S. Tahir \\ Basrah University, \\ Engineering College, \\ Computer Department \\ duniasattar680@gmail.com
}

\begin{abstract}
In this paper, image deblurring and denoising are presented. The used images were blurred either with Gaussian or motion blur and corrupted either by Gaussian noise or by salt \& pepper noise. In our algorithm, the modified fixed-phase iterative algorithm (MFPIA) is used to reduce the blur. Then a discrete wavelet transform is used to divide the image into two parts. The first part represents the approximation coefficients. While the second part represents the detail coefficients, that a noise is removed by using the BayesShrink wavelet thresholding method.
\end{abstract}

Keywords: Image deblurring ; Image denoising; Image Processing; Discrete wavelet transform

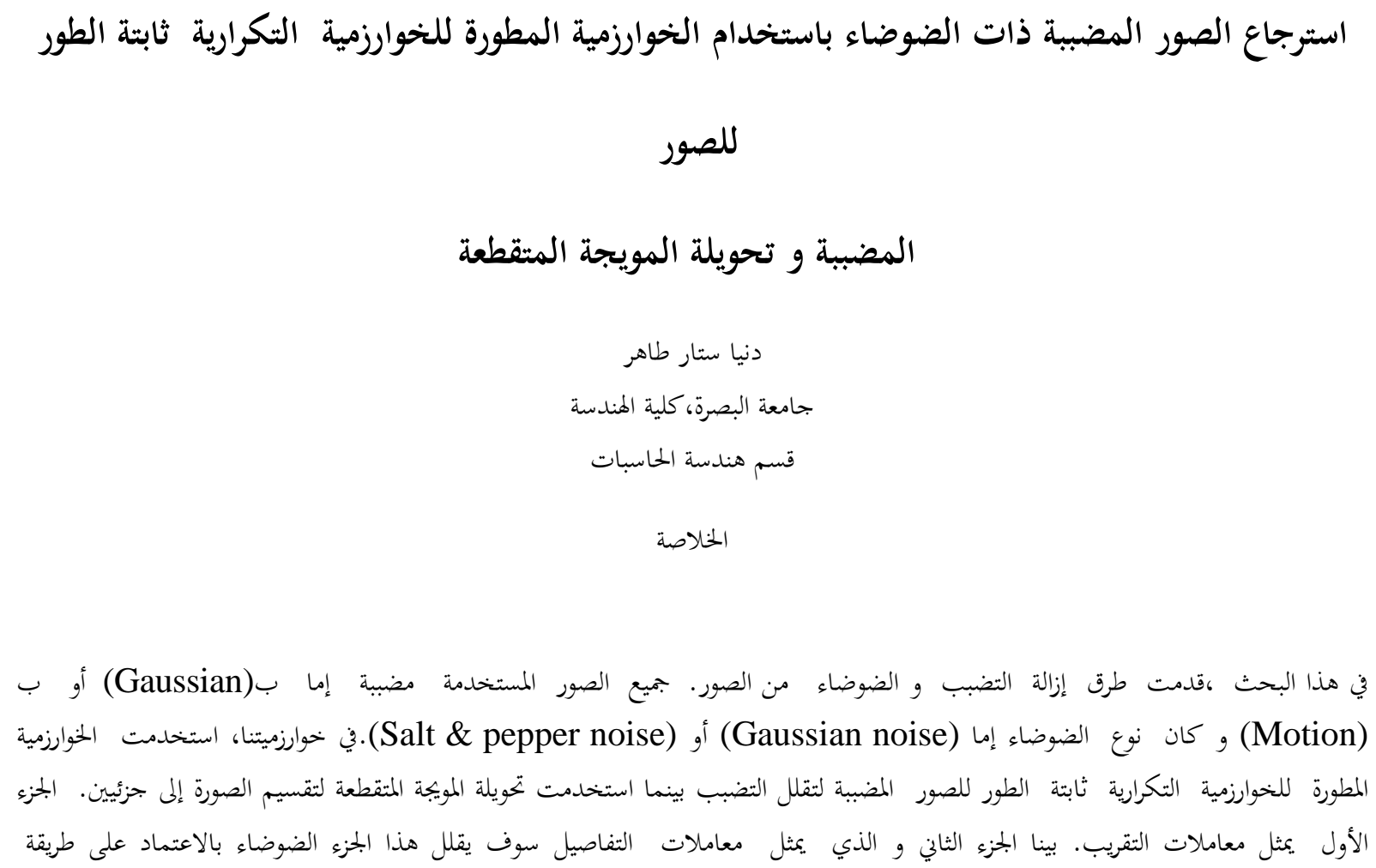

.BayesShrink wavelet thresholding 


\section{Introduction}

Digital image processing deals with many operations such as image compression, image edge enhancement, blurred image restoration, and noise removal from images. Image restoration methods are used to improve the appearance of an image by application of a restoration process that uses a mathematical model for image degradation. It is assumed that the degradation model is known or can be estimated. The idea is to model the degradation process and then apply the inverse process to restore the original image [1].

When noises are found on images, the bad data will be found. It should be noted that signals do not exist without noise while working with data obtained from the real world. Under ideal conditions, this noise may decrease to some negligible levels, while in many practical cases, the signal to noise ratio should be increased to some significant levels, that for all practical purposes denoising is a necessity [2]. Two words must be distinguished , "smoothing " and "denoising". Whereas smoothing removes high frequencies and retains low frequencies, denoising attempts to remove whatever noise present and retain all signal components present regardless of the frequency content of the signal [3].

\section{System Model}

The degradation process model consists of two parts, the degradation function and the noise function. The general model in the spatial domain follows [1]:

$d(r, c)=f(r, c) * h(r, c)+n(r, c)$

$d(r, c)=$ degraded image, where the $*$ denotes the convolution process.

$h(r, c)=$ degradation function(distortion operator), also called the Point-Spread Function $(P S F)$. This function, when convolved with the image, created the distortion.

$f(r, c)=$ original image, so that the purpose of all deblurring and denoising techniques is to separate the convolution product in order to restore $f$ from $d$. $n(r, c)=$ noise function.

\subsection{Blurring Models}

The types of blurring are $[4,5]$ :

\section{i. Gaussian Blur}

The Gaussian blur can be generated by the following filter:

$$
h_{f}(r, c)=e^{-\left(r^{2}+c^{2}\right) / 2 \sigma^{2}}
$$

The number of selected pixels and the deviation sigma ( $\sigma$ ) can be modified in order to control the Gaussian blurring degree on the image.

\section{ii. Motion Blur}

In motion blur, the number of selected pixels to be shifted and the angle 
of shifting $(\theta)$ (shifting direction) can be changed [5].

\subsection{Noise Models}

In the image denoising process, information about the type of noise present in the original image plays a significant role. Typical images are corrupted with noise modeled with either a Gaussian, or salt and pepper distribution $[1,5,6]$ :

\section{i. Gaussian Noise}

Gaussian noise is evenly distributed over the signal. This means that each pixel in the noisy image is the sum of the true pixel value and a random Gaussian distributed noise value. As the name indicates, this type of noise has a Gaussian distribution, which has a bell shaped probability distribution function given by [1]:

$$
Z_{G}(g)=\frac{1}{\sqrt{2 \pi \sigma_{n}^{2}}} e^{-\left((g-\mathrm{m})^{2} / 2 \sigma_{n}^{2}\right)}
$$

where $g$ represents the gray level, $\mathrm{m}$ is the mean or average of the function, and $\sigma$ is the standard deviation of the noise $\left(\sigma_{n}^{2}\right.$ = variance) .

\section{ii. Salt and Pepper Noise}

Salt and pepper noise is an impulse type of noise, which is also referred to as intensity spikes [1]. This is caused generally due to errors in data transmission. It has only two possible values, $a$ and $b$. The probability of each is typically less than 0.1 . The corrupted pixels are set alternatively to the minimum or to the maximum value, giving the image a "salt and pepper" like appearance. Unaffected pixels remain unchanged. For an 8-bit image, the typical value for pepper noise is 0 and for salt noise 255. The salt and pepper noise is generally caused by malfunctioning of pixelelements in the camera sensors, faulty memory location, or time errors in the digitization process.

\section{Wavelet-based Denoising}

\section{Algorithm}

The general wavelet denoising procedure is as follows [7]:

(1) Apply wavelet transform to the noisy image to produce the noisy wavelet coefficients.

(2) Select appropriate threshold limit at each level and specify the type of threshold method (hard or soft thresholding) for best removal of noise.

(3) Inverse wavelet transform of the thresholded waveletcoefficients to obtain a denoised image.

\subsection{Wavelet Thresholding}

Wavelet thresholding is the decomposition of a data or an image into some wavelet coefficients, comparing the detail coefficients with a given threshold value, and shrinking these 
coefficients close to zero to take away the effect of noise in the data.

During thresholding, a wavelet coefficient is compared with a given threshold and is set to zero if its magnitude is less than the threshold; otherwise, it is retained or modified depending on the threshold rule. The choice of a threshold is an important point of interest. It plays a major role in the reduction or removal of noise in images because denoising most frequently produces smoothed images, reducing the sharpness of the image. It is necessary to know about the two general categories of thresholding. They are hard-thresholding and soft- thresholding types [8].

\subsubsection{Hard Thresholding}

The hard-thresholding $\left(T_{H}\right)$ can be defined as [8]:

$$
T_{H}= \begin{cases}x & \text { if }|x|>t \\ 0 & \text { if }|x| \leq t\end{cases}
$$

In hard thresholding, all coefficients whose magnitudes are greater than the selected threshold value remain as they are and the others with magnitudes smaller than or equal are set to zero.

\subsubsection{Soft Thresholding}

The soft-thresholding $\left(T_{S}\right)$ can be defined

$$
T_{S}= \begin{cases}\operatorname{sign}(x)(|x|-t) & \text { if }|x|>t \\ 0 & \text { if }|x| \leq\end{cases}
$$

as [8]:

In soft thresholding, all coefficients whose magnitude is greater than the selected threshold value, a signum function returns the $(+1)$ value when the image coefficient exceeds the preset threshold, returns a(0) when it equals the preset threshold and returns a (-1) when it falls below the threshold. And the others with magnitudes smaller than or equal threshold value are set to zero.

In practice, it is well known that the soft method is much better and yields more visually pleasant images. That is because the hard method is discontinuous and yields abrupt artifacts in the recovered images [9].

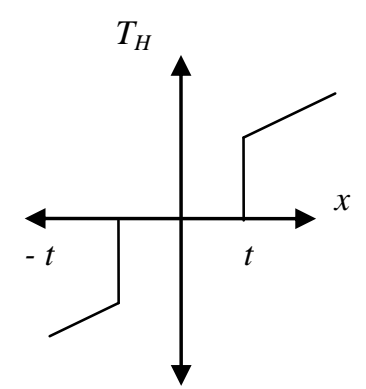

(a)

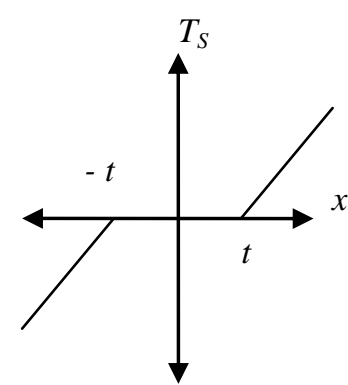

(b)
Figure 1:Shows hard \& soft thresholding: (a) Hard thresholding, and (b) soft thresholding

In Figure 1 which represents the mapping functions of hard and soft thresholdings, while at first sight hard thresholding may seem to be natural, the continuity of soft thresholding has some advantages. It makes algorithms 
mathematically more tractable [10]. Some times, pure noise coefficients may pass the hard threshold and appear as annoying 'blips' in the output. Soft thresholding shrinks these false structures[11, 12, 13].

In fact, since many optimal threshold values were derived for the purpose of soft thresholding, it is a common practice to simply set the optimal hard threshold value to be twice that of the optimal soft threshold .The relationship between the optimal values of hard and soft thresholding can be defined as [6] :

$T_{S} \approx \frac{T_{H}}{2}$

$T_{H} \approx T_{S} \times 2$

So in this paper, soft thresholding will be calculated and then hard thresholding can be calculated by applying Eq. (7) .

\subsection{BayesShrink Wavelet Thresholding}

The goal of this method is to minimize the Bayesian risk, and hence its name, BayesShrink [14]. In BayesShrink the threshold for each subband will be determined. It uses soft thresholding and is subband-dependent, which means that thresholding is done at each band of resolution in the wavelet decomposition. It is smoothless adaptive [14].

The Bayes threshold, $t_{B S}$, is defined as $[1,11,14]$ :

$$
t_{B S}=\sigma_{n}^{2} / \sigma_{f}
$$

where:

$\sigma_{n}^{2}:$ is the noise variance, and $\sigma_{f}^{2}:$ is the image variance without noise. The noise variance $\sigma_{n}^{2}$ is estimated from the subband $\mathrm{HH}$ by the median estimator given as follows:

$\hat{\sigma}=\frac{\operatorname{Median}\left(\left|D_{\mathrm{HH}}(r, c)\right|\right)}{0.6745}$,

where the pixels $D_{\mathrm{HH}}(r, c) \in$ subband $\mathrm{HH}$

From the definition of image corrupted with noise:

$$
d(r, c)=f(r, c)+n(r, c)
$$

Since the noise and the image are independent of each other, it can be stated that:

$$
\sigma_{d}^{2}=\sigma_{f}^{2}+\sigma_{n}^{2}
$$

where $\sigma_{d}^{2}$ can be computed as shown below:

$$
\sigma_{d}^{2}=\frac{1}{R \times C} \sum_{r=1}^{R} \sum_{c=1}^{C} d^{2}(r, c)
$$

he variance of the image, $\sigma_{f}^{2}$ computed as:

$$
\sigma_{f}=\sqrt{\max \left(\sigma_{d}^{2}-\sigma_{n}^{2}, 0\right)}
$$

Knowing $\sigma_{n}^{2}$ and $\sigma_{f}^{2}$ the Bayes threshold can be computed from Eq. (8). Using this threshold, the wavelet coefficients are thresholded at each band.

\section{Modified Fixed Phase Iterative} Algorithm Recovery (MFPIA) of Blurred Images

The Modified Fixed Phase Iterative Algorithm (MFPIA), basically depends on two concepts, one is that introduced by 
Zho Ren Feng and Zhou Hui in the fixed phase iterative recovery algorithm of blurred images which states that the phase spectrum of the original clear image is the same as that for the blurred image [15]. The other concept is presented in the derivation and analysis of Slepin method [16].

In this algorithm, supposes that there is no noise effect $n(r, c)=0$, so that:

$d(r, c)=f(r, c)^{*} h(r, c) \quad 0 \leq(r, c) \leq \mathrm{M}$

The MFPIA algorithm is carried out by implementing the following tasks:

(1) For the first iteration, set $f_{0}(r, c)=$ $d(r, c)$.

(2) Suppose that $p$ is the time of the iteration, for the sake of treatment, $f_{\mathrm{p}}(r, c)$ is needed to be converted to one dimension(1-D) form $f_{\mathrm{p}}(m)$ where $0 \leq m \leq \mathrm{M}^{2}$ by using the vector transform [16].

(3) Convert $f_{\mathrm{p}}(m)$ to its frequency domain representation, i. e., magnitude \& phase by using Fast Fourier Transform (FFT). The length of FFT and Inverse Fast Fourier Transform $\left(\mathrm{FFT}^{-1}\right)$ must be bigger than $2 \mathrm{M}^{2}$ to ensure that the recovery is done perfectly[16]:

$F_{\mathrm{p}}(k)=\operatorname{FFT}\left[f_{\mathrm{p}}(m)\right]$

$D(k)=\mathrm{FFT}[d(m)]$ where $0 \leq k \leq 2 \mathrm{M}^{2}$.

in magnitude and phase forms:

$$
\begin{aligned}
& F_{\mathrm{p}}(k)=\left|F_{\mathrm{p}}(k)\right| \exp \left[j \theta_{F_{p}}(k)\right] \\
& D(k)=|D(k)| \exp \left[j \theta_{D}(k)\right]
\end{aligned}
$$

(4) The phase replacing process should take place here, i. e.,

$$
\theta_{D}(k) \rightarrow \theta_{F_{p}}(k)
$$

Then the new sequence $F_{\mathrm{p}+1}(k)$ is obtained as follows:

$F_{\mathrm{p}+1}(k)=F_{\mathrm{p}}(k) \mid \exp \left[j \theta_{D}(k)\right]$

(5) Now applying $\mathrm{FFT}^{-1}$ to $F_{\mathrm{p}+1}(k), f_{\mathrm{p}+1}(m)$ can be obtained, and since $f_{\mathrm{p}+1}(m)$ is a $2 \mathrm{M}^{2}$ point length it must be truncated into an $\mathbf{M}^{2}$-point length, that is

$$
\hat{f}_{\mathrm{p}+1}(m)= \begin{cases}f_{\mathrm{p}+1}(m) & 0 \leq m \leq \mathrm{M}^{2}-1 \\ 0 & \mathrm{M}^{2} \leq m \leq 2 \mathrm{M}^{2}-1\end{cases}
$$

This is the time truncation process.

(6) Using inverse vector transform the sequence $\hat{f}_{\mathrm{p}+1}(m)$ is transformed to. $\hat{f}_{\mathrm{p}+1}(r, c)$ (7) The derivative operation is performed by using an edge detection operator (e.g. Laplacian operator), thus $\hat{f}_{\mathrm{p}+1}^{\prime}(r, c)$ is obtained.

(8) The restored image at iteration $p+1$ is obtained by

$f_{\mathrm{p}+1}(r, c)=+\hat{f}_{\mathrm{p}+1}(r, c) \hat{f}_{\mathrm{p}+1}^{\prime}(r, c)$

If the restored image quality is not good the iteration is repeated from step2 [16].

\section{The Proposed Algorithm}

The present algorithm will introduced to be able to restore noisy blurred images. It can be manipulate blurring only without noise, because the noise lies at high frequencies. The wavelet transform will be used to distinguish between the low frequency components and high 
frequency components and then it is assumed that there is no effect of noise at low frequencies.

The proposed algorithm will be based on the wavelet transform. Using wavelet transform, images are decomposed into approximation and detail coefficients, where as the detail coefficient is further decomposed into (horizontal,vertical,diagonal)coefficients, where:

- Approximation coefficients $\left(\mathrm{A}_{\mathrm{c}}\right)$ represent the Low-Low subband,

- Horizontal detail coefficients $\left(\mathrm{H}_{\mathrm{c}}\right)$ represent the Low-High subband,

- Vertical detail coefficients $\left(\mathrm{V}_{\mathrm{c}}\right)$ represent the High -Low subband, and

- Diagonal detail coefficients $\left(D_{c}\right)$ represent the High-High subband.

The effect of blurring can be reduced by using the modified fixed phase iterative algorithm MFPIA.While the effect of noise in the image can also be reduced by using BayesShrink method. This idea is realized in our algorithm.

Our algorithm is represented by the following steps:

(1) For the noisy blurred image

$d(r, c)=f(r, c) * h(r, c)+n(r, c)$

$d(r, c)$ : the degraded image

$f(r, c)$ : the original image and $h(r, c)$ : the point spread function (PSF). it is assumed that there is no noise effect in this subband, i. e., $n(r, c)=0$.

A . In the first iteration, after assuming $n(r, c)=0$, set $f_{0}(r, c)=d(r, c)$.

B. Suppose that $\mathrm{p}$ is the time of the iteration, for the sake of treatment $f_{\mathrm{p}}(r, c)$ should be converted to a 1-D form $f_{\mathrm{p}}(m)$ where $0 \leq m \leq \mathrm{M}^{2}$, with $\mathrm{M}=\mathrm{R}=\mathrm{C}$ by using the vector transform [16].

(2)Convert $f_{\mathrm{p}}(m)$ to its frequency domain representation (i. e., magnitude and phase by using FFT). The length of FFT and FFT $^{-1}$ must be bigger than $2 \mathrm{M}^{2}$ to ensure that the recovery is done perfectly. That is:

$$
f_{\mathrm{p}}(k)=\mathrm{FFT}\left(f_{\mathrm{p}}(m)\right)
$$

and

$D(k)=$ FFT $(d(m))$ where $0 \leq k \leq 2 \mathrm{M}^{2}$

Now Eqs. (24) and (25) can be rewritten in the magnitude and phase forms:

$$
\begin{aligned}
& F_{\mathrm{p}}(k)=\left|F_{\mathrm{p}}(k)\right| \exp \left[\mathrm{j} \theta_{F \mathrm{p}}(k)\right] \\
& \text { and }
\end{aligned}
$$

$D(k)=|D(k)| \exp \left[\mathrm{j} \theta_{D}(k)\right]$

(3) The phase replacing process should take place here, i. e.,

$$
\theta_{D}(k) \longrightarrow \theta_{f \mathrm{p}}(k)
$$

Then the new sequence $F_{\mathrm{p}+1}(k)$ is obtained as:

$$
F_{\mathrm{p}+1}(k)=\left|F_{\mathrm{p}}(k)\right| \exp \left[\mathrm{j} \theta_{D}(k)\right]
$$

(4) Now applying $\mathrm{FFT}^{-1}$ to $F_{\mathrm{p}+1}(k), f_{\mathrm{p}+1}(m)$ can be obtained, and since $f_{\mathrm{p}+1}(m)$ is a $2 \mathrm{M}^{2}$-point length it must be truncated into an $\mathrm{M}^{2}$-point length, that is 
$=\hat{f}_{\mathrm{p}+1}(m) \begin{cases}f_{\mathrm{p}+1}(m) & 0 \leq m \leq \mathrm{M}^{2}-1 \\ 0 & \mathrm{M}^{2} \leq m \leq 2 \mathrm{M}^{2}-1\end{cases}$

This is the time truncation process.

(5) Using inverse vector transform, the sequence $\hat{f}_{\mathrm{p}+1}(m)$ is transformed to $\hat{f}_{\mathrm{p}+1}(r, c)$.

(6) The derivative operation is performed by using an edge detection operator (e.g. Laplacian operator), thus $\hat{f}_{\mathrm{p}+1}^{\prime}(r, c)$ is obtained.

(7) The restored image at iteration $p+1$ is obtained by

$$
f_{\mathrm{p}+1}(r, c)=+\hat{f}_{\mathrm{p}+1}(r, c) \hat{f}_{\mathrm{p}+1}^{\prime}(r, c)
$$

If the quality of the restored approximation coefficients of the image is not good the iteration is repeated from step 1B, else the restored image without blurring represents the new approximation coefficients.

(8) Decompose the noisy image into subbands $\left[\mathrm{A}_{\mathrm{c}}, \mathrm{H}_{\mathrm{c}}, \mathrm{V}_{\mathrm{c}}, \mathrm{D}_{\mathrm{c}}\right]$ using the two dimension (2-D) Discrete Wavelet Transform (DWT).

(9) For detail coefficients $\left(\mathrm{H}_{\mathrm{c}}, \mathrm{V}_{\mathrm{c}}, \mathrm{D}_{\mathrm{c}}\right)$, the thresholding values should be found to reduce or remove the effect of noise by using BayesShrink method.

(10) Reconstruct the recovered image by applying the 2-D Inverse Discrete Wavelet Transform (IDWT).

\section{Discussion}

After image processing (restoration), the need to know how much the restored image is compatible to the original image, in other words whether the restoration process is effective or not.

\section{i. Objective Fidelity Criteria}

These criteria are borrowed from digital signal and information theory and provide us with equations that can be used to measure the amount of error in the restored image. The commonly objective measures are the Root Mean Square Error RMS and the Signal to Noise Ratio SNR.

The RMS can be defined as $[6,17,18]$ :

$\mathrm{RMS}=\sqrt{\frac{1}{R \times C} \sum_{r=0}^{R-1} \sum_{c=0}^{C-1}[f(r, c)-\hat{f}(r, c)]^{2}}$

and the SNR can be calculated as $[1,19,20$, 21]:

$\mathrm{SNR}=10 \log \frac{\sum_{r=0}^{R-1} \sum_{c=0}^{C-1}[f(r, c)-d(r, c)]^{2}}{\sum_{r=0}^{R-1} \sum_{c=0}^{C-l}[f(r, c)-\hat{f}(r, c)]^{2}}$

where:

$f(r, c)$ : is the original image, $d(r, c)$ : is the degraded image, and $\hat{f}(r, c)$ : is the restored image.

Another related image quality measure is the Peak Signal to Noise Ratio (PSNR), which is inversely proportional to the RMS, its units are in decibels ( $\mathrm{dB})$ and is formally defined by [6]: 
PSNR $=20 \log _{10}\left[\frac{255}{\mathrm{RMS}}\right]$

where 255 is the maximum pixel value for an 8 bits / pixel gray-scale image. Clearly, when the original image is not known, one cannot rely on the above quantitative fidelity measures. In that case, the assessment of the deblurred and denoised image is done subjectively.

\section{ii. Correlation Factor}

Correlation factor (Cor) measures the similarity between two images and can be defined as [22]:

$C o r=\frac{\sum_{r=1}^{R} \sum_{c=1}^{C}(f(r, c)-\bar{f})(\hat{f}(r, c)-\overline{\hat{f}})}{\sqrt{\left[\sum_{r=1}^{R} \sum_{c=1}^{C}(f(r, c)-\bar{f})^{2}\right]\left[\sum_{r=1}^{R} \sum_{c=1}^{C}(\hat{f}(r, c)-\overline{\hat{f}})^{2}\right]}}$

where:

$f(r, c)$ : is the original image, and $\bar{f}$ : is the mean of the original image $f(r, c)$ that,

$$
\bar{f}=\frac{1}{R \times C} \sum_{r=1}^{R} \sum_{c=1}^{C} f(r, c)
$$

$\hat{f}(r, c)$ : is the restored image, and $\overline{\hat{f}}:$ is the mean of the restored image $\hat{f}(r, c)$ that,

$$
\overline{\hat{f}}=\frac{1}{R \times C} \sum_{r=1}^{R} \sum_{c=1}^{C} \hat{f}(r, c)
$$

" $r, c$ " represents the pixel location $R$ :

height of the two images, and $C$ : width of the two images.

\section{Results}

The application of the presented algorithm will be tested. The images under test will be blurred by two types of blurrings:
1. Gaussian blur.

2. Motion blur.

And also with two types of noise as:

1. Gaussian noise.

2. Salt and pepper noise.

Specific images used are selected with $(512 \times 512)$ pixels spatial resolution and used as the gray-scale type. In our algorithm two level (2-levels), Discrete Wavelet Transform (DWT) is used to decompose the images using Daubechies filter of order (8) denoted as “ db8 ". These filters are found to be appropriate for excellent restoration for all images [8]. The images are degraded with Gaussian blur where the value of $(\sigma)$ will be chosen equal to 2 with the number of selected pixels $=3$ and then with the number of selected pixels $=7$. Motion blur is also tested with the number of the shifted pixels $=4$ and then with the number of the shifted pixels $=8$, both with shifting angle $\theta=0$. While the Gaussian noise is imposed with $\sigma_{n}=10$ and then $\sigma_{n}=15$. The noise density [s] for salt and pepper noise is chosen to be equal to (0.007 and 0.02).
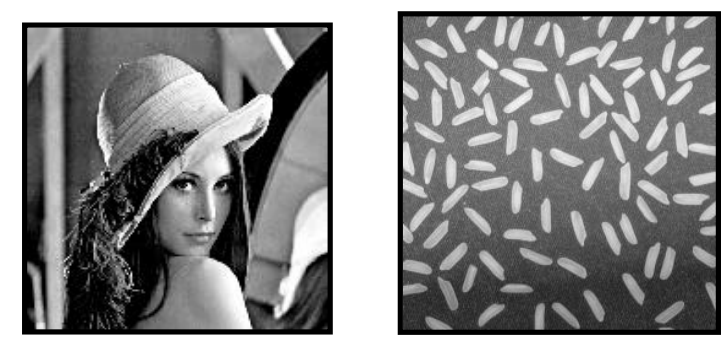

Lena Original image RiceOriginal image

Figure 2: Shows the original images

"Lena" and "Rice" 
The objective fidelity criteria is used to find SNR, PSNR and RMS, the correlation factor is calculated to compare among the images which were corrupted (with noise and blurring) and the original one. Also this factor is calculated to compare between the restored image are "Lena" and "Rice", which are the original images are shown in Figure 2.

These images are restored by using our algorithm and hard, soft thresholding.

In Tables [1, 2, 3 and 4], the first column contains the name of tested images, the second column contains the number of iterations (Iti. no.) needed, the third column contains the type of the added noise, and the forth column is $\left[\sigma_{n}\right]$ or $[\mathbf{s}]$ where:

$\sigma_{n}:$ is the standard deviation of Gaussian noise.

s : is the noise density of salt \& pepper noise.

The fifth column contains the correlation factor for the noisy blurred image and the original one, while the sixth column represents the correlation factor for the restored image and the original one. The columns from seventh to ninth represent the SNR, RMS and PSNR, respectively.

The presented tables, may conclude that our algorithm has good candidates for the restoration of noisy blurred images.
All values have SNR,RMS and PSNR good enough results limited ranges.

\section{Conclusion}

The main advantage of our algorithm is the deblurring process is not needed of PSF deformation. MFPIA using DWT will not be iteratived algorithm because the best results will be obtained for one iteration only. Finally, our algorithm can reduce both noise and blurring from images corrupted with high noise, while all standard methods cannot reduce both noise and blur from images only if the amount of noise is very small. The future work of this paper can be directed to use the wavelet package instead of DWT.

\section{References}

[1] S. E. Umbaugh, "Computer Vision and Image Processing" , Prentice-Hall, Inc., USA , 1998 .

[2] C. H. Vannimenus, "Denoising Images withWavelets",French,2003.

http://www.chez.com/petitspirou/bristol/denoisi ng/report/wavelet.pdf.

[3] C. Taswell, "The What, How, and Why of Wavelet Shrinkage Denoising”, Technical Report, Stanford, CA, 1999.

[4] A. Smirnov and M. Ginzburg, "Iterative Image Restoration",1998.

http://www.cs.technion.ac.il/Labs/Is 1/Project/Pr ojects_done/Vision/Classes/DIP_1998/Iteration _Restoration.

[5] MATLAB R2011a, "Image Processing Toolbox" 
[6] M. Ghazel, "Adaptive Fractal and Wavelet Image Denoising", Ph. D. Thesis Submitted to The Electrical and

Computer Engineering, University of Waterloo, Ontario, Canada, 2004.

[7] Sachin D and Dharmpal D, "Wavelet Based Image Denoising Technique", International Journal of Advanced Computer Science and Applications, Vol. 2, No. 3, March 2011.

[8] MATLAB R2011a, "Wavelet Toolbox". http://www.mathwork.com.

[9] N.Sundaram, “ Wavelet-Based Denoi- sing and Watermarking in Images", Image

Processing Project \# 9.

http://astro.temple.edu/ nithyas/ee740_nithya_r eport9.pdf.

[10] D. L. Donoho, "De-noising by SoftThresholding", IEEE Trans. Infor. Theory, Vol. 41, pp. 613-627, 1995.

[11] D. L. Donoho and I. M. Johnstone, "Adapting to Unknown Smoothness via Wavelet Shrinkage", Journal of American Statistical Association, 90 (432): 1200-1224, December 1995.

[12] A. Antoniadis and J. Bigot, "Wavelet Estimators in Nonparametric Regression: A Comparative Simulation Study", Journal of Statistical Software, Vol. 6, 2001.

[13] R. Rangarajan, R. Venkataramanan and S. Shah, "Image Denoising Using Wavelets ", December 16, 2002.

[14] S. G. Chang, B. Yu and M. Vetterli, "Adaptive Wavelet Thresholding for Image Denoising and Compression", IEEE Trans. Image Processing, Vol. 9, No. 9, pp. 15321546, September 2000.
[15] F. Zhe Ren and H. Zhou, "The FixedPhase Iterative Algorithm Recovery of Blurred Image", ICSP, 2000.

[16] H. A. Jwad, “ A Novel Approach for Restoration of Blurred Images ", M. Sc. Thesis Submitted to The Department of The Computer Engineering, University of Basrah, May 2004

[17] "Digital Image Processing in Natural

Sciences and Medicine".

http://as.ch/DIP/Manual/1-WinterSemes-

ter/DIP-Part-01(6-11).pdf.

[18] J. G. Brankov, J. Djordjeric, M. N. Wernick and N. P. Galatsanos, “ Tomographic Image Reconstruction for System with Partially-Known Blur", Department of Electrical and Computer Engineering, Illinois Institute of Technology, Chicago, USA, 1999. [19] P. G. Nikolas, "Multichannel Regularized Iterative Restoration of Motion Compensated Image Sequences", M. Sc. Thesis Submitted to The Department of Electrical and Computer Engineering, University of Northwestern, March 1995.

[20]X. Miao, “ Image Restoration:Removal of Blur Caused by Uniform Linear Motion", University of California at Berkeley, 2000.

[21] K. K. Aggelos, "Iterative Image Restoration Algorithm”, Optical Engineering, Vol. 28, No. 7, pp. 735-748, July 1989.

[22] H. H. Abdul Zahrah, “ Encryption Using Wavelet Coded Image Data ", M.

Sc. Thesis Submitted to The Department of The Computer Engineering, University of Basrah, June 2004. 
Table 1 The objective results of Lena and Rice images using the proposed algorithm, the blurred Gaussian with values $\sigma=2$ and the number of selected pixels=3, (A) Using hard thresholding, (B) Using soft thresholding.

(A)

\begin{tabular}{|c|c|c|c|c|c|c|c|c|}
\hline $\begin{array}{c}\text { Name } \\
\text { of } \\
\text { image }\end{array}$ & $\begin{array}{l}\text { Iti. } \\
\text { no. }\end{array}$ & $\begin{array}{l}\text { Type } \\
\text { of } \\
\text { noise }\end{array}$ & $\begin{array}{l}\boldsymbol{\sigma}_{n} \\
\text { or s }\end{array}$ & $\begin{array}{c}\text { Correlation } \\
\text { factor of noisy } \\
\text { blurred } \\
\text { Image }\end{array}$ & $\begin{array}{c}\text { Correlation } \\
\text { factor of restored } \\
\text { image }\end{array}$ & $\begin{array}{c}\text { SNR } \\
\text { in } \\
\text { db }\end{array}$ & RMS & $\begin{array}{c}\text { PSNR } \\
\text { in } \\
\text { db }\end{array}$ \\
\hline \multirow{4}{*}{ 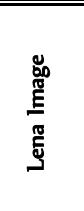 } & 1 & Gaussian & 5 & 0.2844 & 0.9870 & 26.6959 & 6.7110 & 31.5951 \\
\hline & 1 & Gaussian & 10 & 0.2784 & 0.9854 & 26.2329 & 7.0921 & 31.1153 \\
\hline & 1 & Salt\&pepper & 0.002 & 0.2837 & 0.9907 & 28.1423 & 5.6805 & 33.0367 \\
\hline & 1 & Salt\&pepper & 0.02 & 0.2587 & 0.9797 & 24.8731 & 8.3690 & 29.6773 \\
\hline \multirow{4}{*}{ 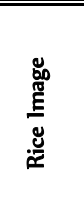 } & 1 & Gaussian & 5 & 0.3505 & 0.9889 & 25.5860 & 6.2400 & 32.1523 \\
\hline & 1 & Gaussian & 10 & 0.3431 & 0.9874 & 25.0732 & 6.6945 & 31.6164 \\
\hline & 1 & Salt\&pepper & 0.002 & 0.3494 & 0.9901 & 26.1095 & 5.9276 & 32.6732 \\
\hline & 1 & Salt\&pepper & 0.02 & 0.3205 & 0.9820 & 23.6122 & 7.9897 & 30.0802 \\
\hline
\end{tabular}

(B)

\begin{tabular}{|c|c|c|c|c|c|c|c|c|}
\hline $\begin{array}{c}\text { Name } \\
\text { of } \\
\text { image }\end{array}$ & $\begin{array}{l}\text { Iti. } \\
\text { no. }\end{array}$ & $\begin{array}{l}\text { Type } \\
\text { of } \\
\text { noise }\end{array}$ & $\begin{array}{l}\boldsymbol{\sigma}_{\boldsymbol{n}} \\
\text { or } \mathrm{s}\end{array}$ & $\begin{array}{c}\text { Correlation } \\
\text { factor of noisy } \\
\text { blurred } \\
\text { Image }\end{array}$ & $\begin{array}{c}\text { Correlation } \\
\text { factor of restored } \\
\text { image }\end{array}$ & $\begin{array}{l}\text { SNR } \\
\text { in } \\
\text { db }\end{array}$ & RMS & $\begin{array}{c}\text { PSNR } \\
\text { in } \\
\text { db }\end{array}$ \\
\hline \multirow{4}{*}{ 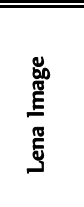 } & 1 & Gaussian & 5 & 0.2844 & 0.9870 & 26.6959 & 6.7110 & 31.5951 \\
\hline & 1 & Gaussian & 10 & 0.2784 & 0.9854 & 26.2329 & 7.0921 & 31.1153 \\
\hline & 1 & Salt\&pepper & 0.002 & 0.2837 & 0.9907 & 28.3484 & 5.5374 & 33.2428 \\
\hline & 1 & Salt\&pepper & 0.02 & "0.2587 & 0.9797 & 24.8731 & 8.3690 & 29.6773 \\
\hline \multirow{4}{*}{ 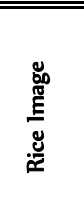 } & 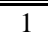 & Gaussian & 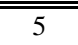 & "0.3505 & "0.9889 & 25.5860 & 6.2400 & 32.1523 \\
\hline & 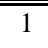 & Gaussian & 10 & "0.3431 & 0.9874 & 25.0732 & "6.6945 & 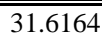 \\
\hline & $\overline{11}$ & Salt\&pepper & 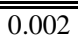 & "0.3494 & "0.9901 & 26.6766 & $\bar{~} 5.5530$ & 33.2403 \\
\hline & $\overline{1}$ & Salt\&pepper & 0.02 & 0.3205 & 0.9820 & 23.6122 & 7.9897 & 30.0802 \\
\hline
\end{tabular}


Table 2 The objective results of Lena and Rice images using the proposed algorithm, the blurred Gaussian with values $\sigma=2$ and the number of selected pixels=7, (A) Using hard thresholding, (B) Using soft thresholding.

(A)

\begin{tabular}{|c|c|c|c|c|c|c|c|c|}
\hline $\begin{array}{l}\text { Name } \\
\text { of } \\
\text { image }\end{array}$ & $\begin{array}{l}\text { Iti. } \\
\text { no. }\end{array}$ & $\begin{array}{l}\text { Type } \\
\text { of } \\
\text { noise }\end{array}$ & $\begin{array}{l}\sigma_{n} \\
\text { or } \mathrm{s}\end{array}$ & $\begin{array}{c}\text { Correlation } \\
\text { factor of noisy } \\
\text { blurred } \\
\text { Image }\end{array}$ & $\begin{array}{c}\text { Correlation } \\
\text { factor of restored } \\
\text { image }\end{array}$ & $\begin{array}{c}\text { SNR } \\
\text { in } \\
\text { db }\end{array}$ & RMS & $\begin{array}{c}\text { PSNR } \\
\text { in } \\
\text { db }\end{array}$ \\
\hline \multirow{4}{*}{ 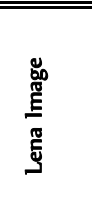 } & 1 & Gaussian & 5 & 0.2800 & 0.9783 & 24.5151 & 8.6843 & 29.3561 \\
\hline & 1 & Gaussian & 10 & 0.2737 & 0.9765 & 24.2176 & 9.0040 & 29.0421 \\
\hline & 1 & Salt\&pepper & 0.002 & 0.2790 & 0.9780 & 24.4584 & 8.7450 & 29.29 .47 \\
\hline & 1 & Salt\&pepper & $\overline{0.02}$ & 0.2538 & 0.9709 & 23.3425 & 10.0492 & 28.0882 \\
\hline \multirow{4}{*}{ 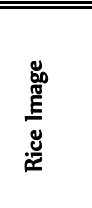 } & 1 & Gaussian & 5 & 0.3458 & 0.9817 & 23.4180 & 8.1527 & 29.9048 \\
\hline & 1 & Gaussian & 10 & 0.3382 & 0.9801 & 23.1055 & 8.4751 & 29.5679 \\
\hline & 1 & Salt\&pepper & 0.002 & 0.3444 & 0.9814 & 23.3470 & 8.2232 & 29.8300 \\
\hline & $\overline{1}$ & Salt\&pepper & 0.02 & 0.3141 & 0.9744 & 22.0962 & 9.5978 & 28.4874 \\
\hline
\end{tabular}

(B)

\begin{tabular}{|c|c|c|c|c|c|c|c|c|}
\hline $\begin{array}{c}\text { Name } \\
\text { of } \\
\text { image }\end{array}$ & $\begin{array}{l}\text { Iti. } \\
\text { no. }\end{array}$ & $\begin{array}{c}\text { Type } \\
\text { of } \\
\text { noise }\end{array}$ & $\begin{array}{l}\boldsymbol{\sigma}_{\boldsymbol{n}} \\
\text { or s }\end{array}$ & $\begin{array}{c}\text { Correlation } \\
\text { factor of noisy } \\
\text { blurred } \\
\text { Image }\end{array}$ & $\begin{array}{c}\text { Correlation } \\
\text { factor of restored } \\
\text { image }\end{array}$ & $\begin{array}{c}\text { SNR } \\
\text { in } \\
\text { db }\end{array}$ & RMS & $\begin{array}{c}\text { PSNR } \\
\text { in } \\
\text { db }\end{array}$ \\
\hline \multirow{4}{*}{ 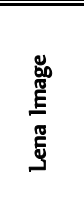 } & 1 & Gaussian & 5 & 0.2800 & 0.9783 & 24.5151 & 8.6843 & 29.3561 \\
\hline & 1 & Gaussian & 10 & 0.2737 & 0.9765 & 24.2176 & 9.0040 & 29.0421 \\
\hline & 1 & Salt\&pepper & 0.002 & 0.2790 & 0.9780 & 24.4584 & 8.7450 & 29.29 .47 \\
\hline & 1 & Salt\&pepper & 0.02 & 0.2538 & 0.9709 & 23.3425 & 10.0492 & 28.0882 \\
\hline \multirow{4}{*}{ 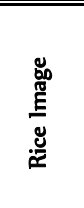 } & 1 & Gaussian & $\overline{5}$ & 0.3458 & 0.9817 & 23.4180 & 8.1527 & 29.9048 \\
\hline & 1 & Gaussian & 10 & 0.3382 & 0.9801 & 23.1055 & 8.4751 & 29.5679 \\
\hline & 1 & Salt\&pepper & 0.002 & 0.3444 & 0.9814 & 23.3470 & 8.2232 & 29.8300 \\
\hline & 1 & Salt\&pepper & 0.02 & 0.3141 & 0.9744 & 22.0962 & 9.5978 & 28.4874 \\
\hline
\end{tabular}


Table 3 The objective results of Lena and Rice images using the proposed algorithm , Motion blurred with the number of the shiftedpixels $=4$, and the shifting angle $\theta=0$. (A)Using hard thresholding, (B) Using soft thresholding.

(A)

\begin{tabular}{|c|c|c|c|c|c|c|c|c|}
\hline $\begin{array}{c}\text { Name } \\
\text { of } \\
\text { image }\end{array}$ & $\begin{array}{l}\text { Iti. } \\
\text { no. }\end{array}$ & $\begin{array}{l}\text { Type } \\
\text { of } \\
\text { noise }\end{array}$ & $\begin{array}{l}\sigma_{n} \\
\text { or } \mathrm{s}\end{array}$ & $\begin{array}{c}\text { Correlation } \\
\text { factor of noisy } \\
\text { blurred } \\
\text { Image }\end{array}$ & $\begin{array}{c}\text { Correlation } \\
\text { factor of restored } \\
\text { image }\end{array}$ & $\begin{array}{c}\text { SNR } \\
\text { in } \\
\text { db }\end{array}$ & RMS & $\begin{array}{c}\text { PSNR } \\
\text { in } \\
\text { db }\end{array}$ \\
\hline \multirow{4}{*}{ 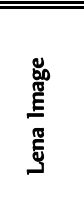 } & 1 & Gaussian & 5 & 0.2841 & 0.9858 & 26.3402 & 6.9929 & 31.2377 \\
\hline & 1 & Gaussian & 10 & 0.2780 & 0.9842 & 25.8994 & 7.3698 & 30.7817 \\
\hline & 1 & Salt\&pepper & 0.002 & 0.2831 & 0.9872 & 26.7776 & 6.6524 & 31.6712 \\
\hline & 1 & Salt\&pepper & $\overline{0.02}$ & 0.2584 & "0.9786 & 24.6361 & 8.6038 & 29.4370 \\
\hline \multirow{4}{*}{ 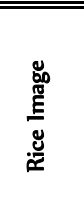 } & 1 & Gaussian & 5 & 0.3500 & 0.9879 & 25.2241 & 6.5648 & 31.7864 \\
\hline & 1 & Gaussian & 10 & 0.3427 & 0.9863 & 24.7396 & 6.9593 & 31.2795 \\
\hline & 1 & Salt\&pepper & 0.002 & 0.3489 & 0.9883 & 25.3949 & 6.4402 & 31.9529 \\
\hline & $\overline{1}$ & Salt\&pepper & 0.02 & 0.3181 & 0.9808 & 23.3260 & 8.2632 & 29.7878 \\
\hline
\end{tabular}

(B)

\begin{tabular}{|c|c|c|c|c|c|c|c|c|}
\hline $\begin{array}{c}\text { Name } \\
\text { of } \\
\text { image }\end{array}$ & $\begin{array}{l}\text { Iti. } \\
\text { no. }\end{array}$ & $\begin{array}{c}\text { Type } \\
\text { of } \\
\text { noise }\end{array}$ & $\begin{array}{l}\boldsymbol{\sigma}_{\boldsymbol{n}} \\
\text { or } \mathrm{s}\end{array}$ & $\begin{array}{c}\text { Correlation } \\
\text { factor of noisy } \\
\text { blurred } \\
\text { Image }\end{array}$ & $\begin{array}{c}\text { Correlation } \\
\text { factor of restored } \\
\text { image }\end{array}$ & $\begin{array}{c}\text { SNR } \\
\text { in } \\
\text { db }\end{array}$ & RMS & $\begin{array}{c}\text { PSNR } \\
\text { in } \\
\text { db }\end{array}$ \\
\hline \multirow{4}{*}{ 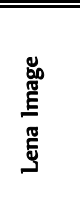 } & 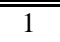 & Gaussian & 5 & 0.2841 & 0.9858 & 26.3402 & 6.9929 & 31.2377 \\
\hline & 1 & Gaussian & 10 & 0.2780 & 0.9842 & 25.8994 & 7.3698 & 30.7817 \\
\hline & 1 & Salt\&pepper & 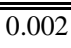 & "0.2831 & 0.9872 & 27.0756 & 6.4280 & 31.9693 \\
\hline & 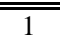 & Salt\&pepper & 0.02 & "0.2584 & 0.9786 & 24.6361 & 8.6038 & 29.4370 \\
\hline \multirow{4}{*}{ 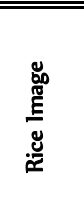 } & 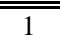 & Gaussian & 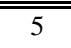 & "0.3500 & 0.9879 & 25.2241 & 6.5648 & 31.7864 \\
\hline & $\bar{~} 1$ & Gaussian & 10 & 0.3427 & "0.9863 & 24.7396 & $\bar{~} 6.9593$ & 31.2795 \\
\hline & 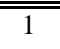 & Salt\&pepper & $\overline{0.002}$ & "0.3489 & 0.9883 & 25.8700 & $\bar{~} 6.0974$ & 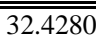 \\
\hline & 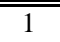 & "Salt\&pepper & 0.02 & 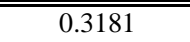 & 0.9808 & 23.3260 & 8.2632 & 29.7878 \\
\hline
\end{tabular}


Table 4 The objective results of Lena and Rice images using the proposed algorithm, Motion blurred with the number of the shiftedpixels $=8$, and the shifting angle $\theta=0$. (A) Using hard thresholding, (B) Using soft thresholding.

(A)

\begin{tabular}{|c|c|c|c|c|c|c|c|c|}
\hline $\begin{array}{c}\text { Name } \\
\text { of } \\
\text { image }\end{array}$ & $\begin{array}{l}\text { Iti. } \\
\text { no. }\end{array}$ & $\begin{array}{c}\text { Type } \\
\text { of } \\
\text { noise }\end{array}$ & $\begin{array}{l}\boldsymbol{\sigma}_{\boldsymbol{n}} \\
\text { or } \mathrm{s}\end{array}$ & $\begin{array}{c}\text { Correlation } \\
\text { factor of noisy } \\
\text { blurred } \\
\text { Image }\end{array}$ & $\begin{array}{c}\text { Correlation } \\
\text { factor of restored } \\
\text { image }\end{array}$ & $\begin{array}{c}\text { SNR } \\
\text { in } \\
\text { db }\end{array}$ & RMS & $\begin{array}{c}\text { PSNR } \\
\text { in } \\
\text { db }\end{array}$ \\
\hline \multirow{4}{*}{ 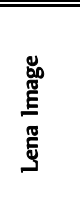 } & 1 & Gaussian & 5 & 0.2784 & 0.9734 & 23.6642 & 9.5813 & 28.5023 \\
\hline & 1 & Gaussian & 10 & 0.2721 & 0.9718 & 23.4355 & 9.8552 & 28.2875 \\
\hline & 1 & Salt\&pepper & 0.002 & 0.2772 & 0.9732 & 23.6282 & 9.6266 & 28.4614 \\
\hline & 1 & Salt\&pepper & 0.02 & 0.2528 & 0.9662 & 22.7194 & 10.7888 & 27.4713 \\
\hline \multirow{4}{*}{ 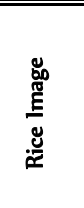 } & 1 & Gaussian & 5 & 0.3446 & 0.9779 & 22.6613 & 8.8981 & 29.1448 \\
\hline & 1 & Gaussian & 10 & 0.3370 & 0.9764 & 22.4064 & 9.1844 & 28.8697 \\
\hline & 1 & Salt\&pepper & 0.002 & 0.3433 & 0.9779 & 22.6516 & 8.9128 & 29.1306 \\
\hline & 1 & Salt\&pepper & 0.02 & 0.3119 & 0.9705 & 21.5167 & 10.2639 & 27.9046 \\
\hline
\end{tabular}

(B)

\begin{tabular}{|c|c|c|c|c|c|c|c|c|}
\hline $\begin{array}{c}\text { Name } \\
\text { of } \\
\text { image }\end{array}$ & $\begin{array}{l}\text { Iti. } \\
\text { no. }\end{array}$ & $\begin{array}{c}\text { Type } \\
\text { of } \\
\text { noise }\end{array}$ & $\begin{array}{l}\boldsymbol{\sigma}_{\boldsymbol{n}} \\
\text { or s }\end{array}$ & $\begin{array}{c}\text { Correlation } \\
\text { factor of noisy } \\
\text { blurred } \\
\text { Image }\end{array}$ & $\begin{array}{c}\text { Correlation } \\
\text { factor of restored } \\
\text { image }\end{array}$ & $\begin{array}{c}\text { SNR } \\
\text { in } \\
\text { db }\end{array}$ & RMS & $\begin{array}{c}\text { PSNR } \\
\text { in } \\
\text { db }\end{array}$ \\
\hline \multirow{4}{*}{ 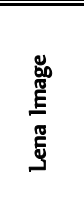 } & 1 & Gaussian & 5 & 0.2784 & 0.9734 & 23.6642 & 9.5813 & 28.5023 \\
\hline & 1 & Gaussian & 10 & 0.2721 & 0.9718 & 23.4355 & 9.8552 & 28.2875 \\
\hline & 1 & Salt\&pepper & 0.002 & 0.2772 & 0.9732 & 23.6282 & 9.6266 & 28.4614 \\
\hline & 1 & Salt\&pepper & 0.02 & 0.2528 & 0.9662 & 22.7194 & $\begin{array}{l}10.7888 \\
\end{array}$ & 27.4713 \\
\hline \multirow{4}{*}{ 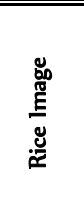 } & 1 & Gaussian & 5 & 0.3446 & 0.9779 & 22.6613 & 8.8981 & 29.1448 \\
\hline & 1 & Gaussian & 10 & 0.3370 & 0.9764 & 22.4064 & 9.1844 & 28.8697 \\
\hline & 1 & Salt\&pepper & 0.002 & 0.3433 & 0.9779 & 22.7541 & 8.8083 & 29.2330 \\
\hline & 1 & Salt\&pepper & 0.02 & 0.3119 & 0.9705 & 21.5167 & 10.2639 & 27.9046 \\
\hline
\end{tabular}

